\title{
Kinetic instability of ion acoustic mode in permeating plasmas
}

\author{
J. Vranjes, ${ }^{1}$ S. Poedts, ${ }^{1}$ and Zahida Ehsan ${ }^{2}$ \\ ${ }^{1}$ Center for Plasma Astrophysics and Leuven Mathematical Modeling and Computational Science Center \\ (LMCC), K. U. Leuven, Celestijnenlaan 200B, 3001 Leuven, Belgium \\ ${ }^{2}$ Salam Chair in Department of Physics, GC University, Lahore 54000, Pakistan \\ and Plasma Physics Group, Blackett Laboratory, Imperial College, London SW7 2AZ, United Kingdom
}

(Received 30 April 2009; accepted 17 June 2009; published online 7 July 2009)

\begin{abstract}
In plasmas with electron drift (current) relative to static ions, the ion acoustic wave is subject to the kinetic instability which takes place if the directed electron speed exceeds the ion acoustic speed. The instability threshold becomes different in the case of one quasineutral electron-ion plasma propagating through another static quasineutral (target) plasma. The threshold velocity of the propagating plasma may be well below the ion acoustic speed of the static plasma. Such a currentless instability may frequently be expected in space and astrophysical plasmas. (C) 2009
\end{abstract} American Institute of Physics. [DOI: 10.1063/1.3174435]

In an unmagnetized plasma with a macroscopic velocity of the electron component $\vec{v}_{e 0}=v_{e 0} \vec{e}_{z}$ relative to static singly charged ions, a kinetic instability of the ion acoustic (IA) mode sets in, described by the growth rate ${ }^{1}$

$$
\omega_{i}=\left(\frac{\pi}{8}\right)^{1 / 2} k c_{s}\left[\left(\frac{m_{e}}{m_{i}}\right)^{1 / 2}\left(\frac{v_{e 0}}{c_{s}}-1\right)-\tau^{3 / 2} \exp \left(-\frac{\tau}{2}\right)\right] .
$$

Here, $c_{s}=\left(\kappa T_{e} / m_{i}\right)^{1 / 2}$ is the ion sound speed and $\tau=T_{e} / T_{i}$. The necessary condition

$$
v_{e 0}>c_{s}\left[1+\left(m_{i} / m_{e}\right)^{1 / 2} \tau^{3 / 2} \exp (-\tau / 2)\right]
$$

may imply a rather high electron current magnitude. A similar description of the acoustic mode and the growth rate may be obtained also in the case of a magnetized plasma ${ }^{2}$ with the equilibrium magnetic field $\vec{B}_{0}=b_{0} \vec{e}_{z}$ and for perturbations propagating in the direction of the electron current $\vec{v}_{e 0}$ $=v_{e 0} \vec{e}_{z}$ that is parallel to the magnetic field vector. The instability described by Eq. (1) is a purely kinetic, collisionless electron inertia effect. Its two-fluid counterpart, ${ }^{3,4}$ however, is a strictly electron-collision effect.

The instability in Eq. (1) appears due to the Doppler shift term (the one containing $v_{e 0}$ ), which may determine the sign of the growth rate. However, a much lower instability threshold may be obtained in the case of two interpenetrating (permeating) plasmas. This is then a currentless instability, where now the Doppler shift in ions appears to play the main role. This will be demonstrated in the forthcoming text. Such permeating plasmas can be created in laboratory conditions, while in space this happens to be a rather frequent situation, for example, in the case of colliding astrophysical clouds, in the propagation of plasmas originating from the explosions of novae and supernovae and moving through the surrounding plasmas, and also in the case of solar and stellar winds. In the case of the solar wind, one electron-ion quasineutral component is generated mainly in the polar regions (fast wind), while the other electron-ion quasineutral component (slow wind) originates from lower latitudes, yet at large distances from the origin there may be overlapping between the two. A most obvious example of such permeating plasmas is seen also in the solar atmosphere where plasma streams from lower layers are seen continuously propagating along magnetic field structures toward and through the solar corona. From the text and the results that follow it will become clear that the present general study of two interpenetrating plasmas comprises also, as special cases, various examples of plasmas containing some additional (electron or ion) species.

In all of these examples we have one fast flowing plasma whose parameters we shall denote by the subscript $f$ and one slow moving (target) plasma with the parameters denoted by the subscript $s$. In fact, the target plasma can be nonmoving (or "static," hence the same index $s$ ), or if it does move with some constant velocity $\vec{v}_{s 0}=v_{s 0} \vec{e}_{z}$, the corresponding equations for the whole combined plasma system can conveniently be written in the coordinate frame moving with this same velocity. Therefore, in the forthcoming text the corresponding velocity of the other component $\vec{v}_{f 0}=v_{f 0} \vec{e}_{z}$ will be used to describe just the difference between the two.

In the case of singly charged ions, the quasineutrality conditions for the two plasmas in the equilibrium read

$$
n_{f i 0}=n_{f e 0}=n_{f 0}, \quad n_{s i 0}=n_{s e 0}=n_{s 0} .
$$

In general $n_{s 0} \neq n_{f 0}$, and the same holds for the temperatures of the two separate quasineutral plasmas $T_{f e} \neq T_{s e}, T_{f i} \neq T_{s i}$.

We use the plasma distribution function for the species $j$,

$$
f_{j 0}=\frac{n_{j 0}}{(2 \pi)^{3 / 2} v_{T j}^{3}} \exp \left\{-\frac{1}{2 v_{T j}^{2}}\left[v_{x}^{2}+v_{y}^{2}+\left(v_{z}-v_{j 0}\right)^{2}\right]\right\},
$$

where $n_{j 0}=$ const and $v_{T j}^{2}=\kappa T_{j} / m_{j}$. From the linearized Boltzmann kinetic equation for the perturbed distribution function

$$
\frac{\partial f_{j 1}}{\partial t}+\vec{v} \frac{\partial f_{j 1}}{\partial \vec{r}}+\frac{q_{j}}{m_{j}}\left(\vec{E}_{1} \frac{\partial f_{j 0}}{\partial \vec{v}}+\vec{v} \times \vec{B}_{0} \frac{\partial f_{j 1}}{\partial \vec{v}}\right)=0
$$


and for longitudinal electrostatic perturbations $\sim \exp (-i \omega$ $+i k z$ ) we obtain

$$
f_{j 1}=-\frac{i q_{j}}{m_{j}} \frac{\vec{E}_{1}}{\omega-\vec{k} \vec{v}} \frac{\partial f_{j 0}}{\partial \vec{v}} .
$$

Further, using the Ampére law

$$
0=\mu_{0} \vec{j}+\frac{1}{c^{2}} \frac{\partial \vec{E}}{\partial t}
$$

and the expression for the macroscopic current

$$
\vec{j}=\sum_{j} q_{j} \int \vec{v} f_{j} d^{3} \vec{v},
$$

we arrive at

$$
1=\sum_{j} \frac{\omega_{p j}^{2}}{n_{j 0} \omega v_{T j}^{2}} \int_{-\infty}^{\infty} \frac{v_{z}\left(v_{z}-v_{j 0}\right)}{\omega-k v_{z}} f_{j 0} d v_{x} d v_{y} d v_{z} .
$$

The remaining integrations in Eq. (5) are straightforward and the final result is

$$
1+\sum_{j} \frac{1}{k^{2} \lambda_{d j}^{2}}\left[1-Z\left(b_{j}\right)\right]=0
$$

Here, $b_{j}=\left(\omega-k v_{j 0}\right) /\left(k v_{T j}\right), \lambda_{d j}=v_{T j} / \omega_{p j}, \omega_{p j}^{2}=q_{j}^{2} n_{j 0} /\left(\varepsilon_{0} m_{j}\right)$, and

$$
Z\left(b_{j}\right)=\frac{b_{j}}{(2 \pi)^{1 / 2}} \int_{c} \frac{\exp \left(-\zeta^{2} / 2\right)}{b_{j}-\zeta} d \zeta
$$

is the plasma dispersion function with the integration over the Landau contour $c$. The dispersion equation (6) describes the plasma (Langmuir) and the acoustic oscillations.

In the case $v_{s j 0}=0, v_{f j 0}=v_{f 0}$, expanding Eq. (6) in the IA frequency range on conditions

$$
k v_{T s i} \ll|\omega| \ll k v_{T s e}, \quad\left|\omega-k v_{f 0}\right| \ll k v_{T f e}, k v_{T f i},
$$

where $v_{T s j}^{2}=\kappa T_{s j} / m_{j}, v_{T f j}^{2}=\kappa T_{f j} / m_{j}$, we obtain the dispersion equation for the acoustic wave

$$
\begin{aligned}
\Delta(k, \omega) \equiv & +\frac{1}{k^{2} \lambda_{d}^{2}}-\frac{\omega_{p s i}^{2}}{\omega^{2}}-\frac{3 k^{2} v_{T s i}^{2} \omega_{p s i}^{2}}{\omega^{4}} \\
& +i\left(\frac{\pi}{2}\right)^{1 / 2}[\underbrace{\frac{\omega \omega_{p s e}^{2}}{k^{3} v_{T s e}^{3}}}_{c}+\left(\omega-k v_{f 0}\right) \\
& \left.\times(\underbrace{\frac{\omega_{p f e}^{2}}{k^{3} v_{T f e}^{3}}}_{a}+\underbrace{\frac{\omega_{p f i}^{2}}{k^{3} v_{T f i}^{3}}}_{b})+\frac{\omega \omega_{p s i}^{2}}{k^{3} v_{T s i}^{3}} \exp \left(-\frac{\omega^{2}}{2 k^{2} v_{T s i}^{2}}\right)\right]
\end{aligned}
$$

$=0$.
Here, $1 / \lambda_{d}^{2}=1 / \lambda_{d s e}^{2}+1 / \lambda_{d f e}^{2}+1 / \lambda_{d f i}^{2}, \lambda_{d s e}=v_{T s e} / \omega_{p s e}$, etc. In view of the condition (7) the inertia of the IA mode is provided mainly by the ions of the static (target) plasma.

In standard electron-ion plasma with streaming electrons and for $T_{i} \ll T_{e}$ it yields

$$
\begin{gathered}
1+\frac{1}{\lambda_{d e}^{2} k^{2}}-\frac{\omega_{p i}^{2}}{\omega^{2}}+i(\pi / 2)^{1 / 2}\left\{\frac{\omega_{p e}^{2}\left(\omega-k v_{e 0}\right)}{k^{3} v_{T e}^{3}}\right. \\
\left.+\frac{\omega_{p i}^{2} \omega}{k^{3} v_{T i}^{3}} \exp \left[-\omega^{2} /\left(2 k^{2} v_{T i}^{2}\right)\right]\right\}=0 .
\end{gathered}
$$

From this one can further obtain the growth rate (1) and the frequency $\omega^{2} \simeq k^{2} c_{s}^{2}$.

The only terms in Eq. (8) that may produce a growing mode are those with the Doppler shift, the two other terms in the imaginary part always give the Landau damping. It is easily seen that the electron part in the Doppler-shifted term is usually negligible because $a / b=\left(T_{f i} / T_{f e}\right)^{3 / 2}\left(m_{e} / m_{i}\right)^{1 / 2} \ll 1$. Assuming also that

$$
\begin{aligned}
c /\left[b\left(\omega-k v_{f 0}\right)\right]= & \left|\omega /\left(\omega-k v_{f 0}\right)\right|\left(n_{s 0} / n_{f 0}\right) \\
& \times\left(T_{f i} / T_{s e}\right)^{3 / 2}\left(m_{e} / m_{i}\right)^{1 / 2} \ll 1,
\end{aligned}
$$

the dispersion equation becomes

$$
\begin{aligned}
& 1+ \frac{1}{\lambda_{d}^{2} k^{2}}-\frac{\omega_{p s i}^{2}}{\omega^{2}}-\frac{3 k^{2} v_{T s i}^{2} \omega_{p s i}^{2}}{\omega^{4}}+i(\pi / 2)^{1 / 2}\left\{\frac{\omega_{p f i}^{2}\left(\omega-k v_{f 0}\right)}{k^{3} v_{T f i}^{3}}\right. \\
&\left.+\frac{\omega_{p s i}^{2} \omega}{k^{3} v_{T s i}^{3}} \exp \left[-\omega^{2} /\left(2 k^{2} v_{T s i}^{2}\right)\right]\right\}=0 .
\end{aligned}
$$

The frequency of the IA wave which follows from this is

$$
\omega_{r}^{2}=\left(k^{2} \lambda_{d}^{2} \omega_{p s i}^{2} / 2\right)\left[1+\left(1+12 \lambda_{s i}^{2} / \lambda_{d}^{2}\right)^{1 / 2}\right] .
$$

For an ordinary electron-ion plasma with $s$-species only, it takes the shape $\omega_{r}^{2}=\left(k c_{s}^{2} / 2\right)\left[1+\left(1+12 T_{s i} / T_{s e}\right)^{1 / 2}\right]$.

The growth rate from Eq. (10), $\omega_{i} \simeq-\operatorname{Im} \Delta\left(k, \omega_{r}\right)$ $/[\partial(\operatorname{Re} \Delta) / \partial \omega]_{\omega \simeq \omega_{r}}$, becomes

$$
\begin{aligned}
\omega_{i}= & -\left(\frac{\pi}{8}\right)^{1 / 2} \frac{\omega_{r}^{3}}{k^{2} v_{T f i}^{2}\left(1+6 k^{2} v_{T s i}^{2} / \omega_{r}^{2}\right)}\left\{\frac{\omega_{r}-k v_{f 0}}{k v_{T f i}} \frac{n_{f 0}}{n_{s 0}}\right. \\
& \left.+\frac{\omega_{r}}{k v_{T s i}} \frac{T_{f i}}{T_{s i}} \exp \left[-\omega_{r}^{2} /\left(2 k^{2} v_{T s i}^{2}\right)\right]\right\} .
\end{aligned}
$$

In the limit $T_{f e} \gg T_{f i}, T_{s e} \gg T_{s i}$, and setting $\omega_{r} \simeq k c_{s e}$, the growth rate becomes

$$
\begin{aligned}
\omega_{i}= & \left(\frac{\pi}{8}\right)^{1 / 2} k c_{s e}\left(\frac{T_{s e}}{T_{f i}}\right)^{3 / 2}\left\{\left(\frac{v_{f 0}}{c_{s e}}-1\right) \frac{n_{f 0}}{n_{s 0}}\right. \\
& \left.-\left(\frac{T_{f i}}{T_{s i}}\right)^{3 / 2} \exp \left[-T_{s e} /\left(2 T_{s i}\right)\right]\right\} .
\end{aligned}
$$

From this the instability threshold is

$$
v_{f 0}>c_{s e}\left[1+\frac{n_{s 0}}{n_{f 0}}\left(\frac{T_{f i}}{T_{s i}}\right)^{3 / 2} \exp \left(-\frac{T_{s e}}{2 T_{s i}}\right)\right] .
$$

Here, $c_{s e}^{2}=\kappa T_{s e} / m_{i}$. Equation (14) has remarkable properties. It is seen that here [as compared to Eq. (2)] the first temperature ratio contains only the two ion temperatures, and in addition the electron-ion mass ratio vanishes. In fact, the 


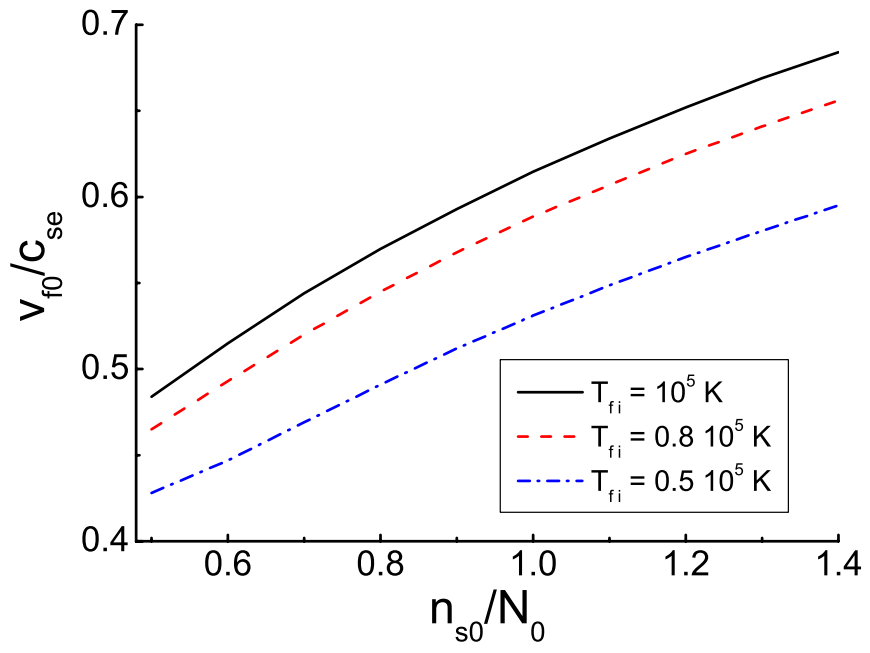

FIG. 1. (Color online) The critical (threshold) values of the flowing plasma velocity for the ion-acoustic wave instability in terms of the number density of the static (target) plasma. Here $c_{s e}^{2}=\kappa T_{s e} / m_{i}, N_{0}=10^{17} \mathrm{~m}^{-3}$.

mass ratio can appear, yet in the present case it would contain the two ion masses (that are taken as equal). For the singly charged two ion species, instead of $n_{s 0} / n_{f 0}$ in Eq. (14) we would have $\left(n_{s 0} / n_{f 0}\right)\left(m_{f i} / m_{s i}\right)$. Consequently, in the present case the instability threshold may become considerably lower. This is particularly valid for a flowing plasma being accelerated into a less dense but hotter static target plasma. One example of that kind may be the plasma from the lower solar atmosphere propagating through hot upper layers (e.g., spicules, and flows along magnetic loops in general).

However, in general case the threshold velocity $v_{f 0}$ for the currentless instability may in fact be below the sound velocity of the target plasma. As an example, the full Eq. (9) is solved numerically by taking the wavelength $\lambda=0.3 \mathrm{~m}$, and $T_{s e}=10^{5} \mathrm{~K}, T_{s i}=10^{3} \mathrm{~K}, n_{f 0}=10^{17} \mathrm{~m}^{-3}$, and $n_{s 0}$ is varied in the range shown in Fig. 1. The critical value of the flowing plasma velocity is shown for several values of the temperature of the flowing plasma. For the velocities above the given lines the IA mode becomes growing. For these parameters, in Eq. (11) the term $\lambda_{s i}^{2} / \lambda_{d}^{2}=0.07$ and also $\lambda_{d} \omega_{p s i}=0.38 c_{s e}$. Therefore, contrary to Eq. (1) or Eq. (14) the instability threshold (cf. Fig. 1) is in fact well below the IA speed of the static (target) plasma. For lower $T_{f i}$ the instability threshold is reduced because of a lower Landau damping by the flowing ions.

Note that for the used parameters, $a / b \ll 1$ but $c / b$ is on the order of unity or larger. Yet, both the mode frequency and the growth rate are well analytically described by Eqs. (11) and (12), respectively.

Instead of condition (7), the expansion in Eq. (6) could be done using the following limits:

$$
k v_{T s i} \ll|\omega| \ll k v_{T s e}, \quad k v_{T f i} \ll\left|\omega-k v_{f 0}\right| \ll k v_{T f e} .
$$

The dispersion equation in this case becomes

$$
\begin{aligned}
\Delta(\omega, k)= & 1+\frac{1}{k^{2} \lambda_{d s e}^{2}}+\frac{1}{k^{2} \lambda_{d f e}^{2}}-\frac{1}{k^{2} \lambda_{d s i}^{2}} \frac{1}{b_{s i}^{2}}\left(1+\frac{3}{b_{s i}^{2}}\right) \\
& -\frac{1}{k^{2} \lambda_{d f i}^{2}} \frac{1}{b_{f i}^{2}}\left(1+\frac{3}{b_{f i}^{2}}\right) \\
& +i\left(\frac{\pi}{2}\right)^{2}\left(\frac{b_{s e}}{k^{2} \lambda_{d s e}^{2}}+\frac{b_{f e}}{k^{2} \lambda_{d f e}^{2}}\right)+i\left(\frac{\pi}{2}\right)^{2} \\
& \times\left[\frac{b_{s i}}{k^{2} \lambda_{d s i}^{2}} \exp \left(-b_{s i}^{2} / 2\right)+\frac{b_{f i}}{k^{2} \lambda_{d f i}^{2}} \exp \left(-b_{f i}^{2} / 2\right)\right] \\
= & 0 .
\end{aligned}
$$

Here, $b_{f j}=\left(\omega-k v_{f 0}\right) / k v_{T f j}$ and $b_{s j}=\omega / k v_{T s j}$. It is seen that as compared to Eq. (10), in the present case the mode is determined by both ion species, while the growth rate is an interplay between the fast ions and fast electrons. The growth rate can easily be discussed in the limit $T_{f i}=T_{s i}, T_{s e}=T_{f e}$ and for both plasmas with the same density $n_{0}$. In this case the imaginary part of Eq. (16) becomes

$$
\begin{aligned}
\operatorname{Im} \Delta= & \left(\frac{\pi}{2}\right)^{2}\left\{\frac{\omega_{p e}^{2}}{k^{3} v_{T e}^{3}}+\frac{\omega_{p i}^{2}}{k^{3} v_{T i}^{3}} \exp \left[-\omega^{2} /\left(2 k^{2} v_{T i}^{2}\right)\right]\right\} \\
& \times\left(2 \omega-k v_{f 0}\right) .
\end{aligned}
$$

If in addition $\tau \equiv T_{e} / T_{i} \gg 1$, the ratio of the electron and ion terms gives the function $f(\tau)=\left(m_{e} / m_{i}\right)^{1 / 2} \tau^{-3 / 2} \exp (\tau / 2)$. For an electron-proton plasma we have $f(\tau)>1$ if $\tau \geq 16$, and only in this limit the growth rate is determined by the electron flow term. Otherwise, the IA instability is driven by the ion Doppler shift.

The cases of dominant ion (or electron) terms discussed above are equivalent to studying also electron-ion plasmas containing an additional ion (or electron species) ${ }^{5-8}$ As special cases, these include also pair ion, ${ }^{9-12}$ negative ion, ${ }^{13}$ electron-positron-ion plasmas, ${ }^{14,15}$ and also dusty plasmas without ${ }^{16}$ and with oppositely charged grains. ${ }^{17}$ Such plasmas have been extensively studied in the recent past. The general streaming kinetic instability presented above is directly applicable to all these plasmas as special cases.

The results presented here are obtained in the framework of the projects FWO-Vlaanderen (Grant No. G.0304.07), ESA Prodex 9 (Grant No. C 90347), and K. U. Leuven (Grant No. GOA/2009-009). Financial support by the European Commission through the SOLAIRE Network (Grant No. MTRN-CT-2006-035484) is gratefully acknowledged.

${ }^{1}$ R. J. Goldston and P. H. Rutherford, Introduction to Plasma Physics (IOP, Bristol, 1995).

${ }^{2}$ A. Y. Wong, Phys. Fluids 9, 1261 (1966).

${ }^{3}$ J. Vranjes, M. Y. Tanaka, and S. Poedts, Phys. Plasmas 13, 122103 (2006).

${ }^{4}$ J. Vranjes, B. P. Pandey, and S. Poedts, Phys. Plasmas 14, 032106 (2007).

${ }^{5}$ H. X. Vu, J. M. Wallace, and B. Bezzerides, Phys. Plasmas 1, 3542 (1994).

${ }^{6}$ J. Vranjes, D. Petrovic, B. P. Pandey, and S. Poedts, Phys. Plasmas 15, 072104 (2008).

${ }^{7}$ R. Sabry, W. M. Moslem, and P. K. Shukla, Phys. Plasmas 16, 032302 (2009).

${ }^{8}$ W. D. Jones, A. Lee, S. M. Gleman, and H. J. Doucet, Phys. Rev. Lett. 35, 1349 (1975).

${ }^{9}$ W. Oohara and R. Hatakeyama, Phys. Rev. Lett. 91, 205005 (2003). 
${ }^{10}$ J. Vranjes and S. Poedts, Plasma Sources Sci. Technol. 14, 485 (2005).

${ }^{11}$ H. Saleem, Phys. Plasmas 14, 014505 (2007).

${ }^{12}$ I. Kourakis, A. Esfandyari-Kalejahi, M. Medhipoor, and P. K. Shukla, Phys. Plasmas 13, 052117 (2006).

${ }^{13}$ B. Song, N. D’Angelo, and R. L. Merlino, Phys. Fluids B 3, 284 (1991).
${ }^{14}$ S. Ali, W. M. Moslem, P. K. Shukla, and R. Schlickeiser, Phys. Plasmas 14, 082307 (2007).

${ }^{15}$ S. A. Shan and H. Saleem, Phys. Plasmas 16, 022111 (2009).

${ }^{16}$ A. A. Mamun and P. K. Shukla, Phys. Plasmas 7, 4412 (2000).

${ }^{17}$ N. D’Angelo, Planet. Space Sci. 49, 1251 (2001). 